\title{
ERRATUM
}

\section{Intuitive calculation of the relativistic Rayleigh-Taylor instability linear growth rate-ERRATUM}

\section{ANTOINE BRET}

ETSI Industriales, Universidad de Castilla-La Mancha, Ciudad Real, Spain

(Laser and Particle Beams (2011), 29, 255-257; doi:10.1017/S0263034611000231)

Due to a production error the secondary affiliation for Dr. Bret was deleted. Below please find the missing affiliation:

Instituto de Investigaciones Energéticas y Aplicaciones Industriales, Campus Universitario de Ciudad Real, 13071 Ciudad Real, Spain

Also note that the e-mail address is incorrect. The correct e-mail address is as follows:

antoineclaude.bret@uclm.es 